\title{
Direct measurement of intense sub-4-fs pulses in a gas target by 3rd-harmonic dispersion-scan
}

\author{
Helder Crespo ${ }^{1,2,},{ }^{*}$ Miguel Canhota ${ }^{1}$, Tobias Witting ${ }^{2,3}$, and John Tisch $^{2}$ \\ ${ }^{1}$ IFIMUP-IN and Departamento de Física e Astronomia, Faculdade de Ciências, Universidade do \\ Porto, R. do Campo Alegre 687, 4169-007 Porto, Portugal \\ ${ }^{2}$ Blackett Laboratory, Imperial College, London SW7 2AZ, UK \\ ${ }^{3}$ Current address: Max-Born-Institute for Nonlinear Optics and Short Pulse Spectroscopy, Max-Born- \\ Straße 2 A, 12489 Berlin, Germany
}

\begin{abstract}
We demonstrate the on-target temporal characterization of sub4-fs pulses by third-harmonic dispersion-scan, using a minimal in-line setup where a gas target optimized for high-harmonic generation doubles as nonlinear medium, obtaining excellent agreement with independent SEA-F-SPIDER measurements.
\end{abstract}

\section{Introduction}

Intense carrier-envelope phase stable few-cycle light pulses are a key tool for attosecond science. Their temporal characterization is paramount for advanced applications and for optimizing the pulse generation itself, but can be very challenging due to the ultra-broad bandwidths of the pulses and their extreme sensitivity to dispersion. Techniques such as spectral phase interferometry for direct electric-field reconstruction (SPIDER), in its spatially encoded arrangement (SEA)-SPIDER [1] implementation, have been successfully used to measure sub-4-fs pulses and are an important part of attosecond technology today [2]. More recently, dispersion-scan (d-scan) [3] has proven to be a robust method for accessing and controlling near-single-cycle pulses [4], with durations down to 1.04-cycles (2.2 fs) already demonstrated [5]. Experimentally, the d-scan technique is based on measuring the spectrum of a nonlinear signal (e.g., second-harmonic generation) as a function of dispersion. The resulting $2 \mathrm{D}$ trace encodes information about the spectral phase of the pulses, which is then retrieved with an optimization algorithm [3]. The ability to measure the pulse temporal profile of intense few-cycle pulses, online and on-target, should provide invaluable information for the quantification of time-dependent dynamics within the same experiment. However, pulse measurement is usually performed off-target using independent setups, and even though complete pulse characterization can be performed with attosecond streaking on a dedicated target (see, e.g., [2]), simpler all-optical methods capable of simultaneously characterizing intense laser pulses within a strong-field or quantum dynamics experiment have been lacking. A variant of SPIDER was used to measure weak (150 nJ) 5 fs pulses in a gas ionized by a long pump pulse [6], but the required low intensities preclude accessing the strong-field regime. Intense few-cycle pulse

*Corresponding author: hcrespo@fc.up.pt 
measurement has been performed in-situ in an attosecond transient absorption spectroscopy experiment, which involved using attosecond extreme-ultraviolet (XUV) pulses together with fs laser pulses interacting in a helium gas cell in a pump-probe scheme to extract the time-delay-dependent phase shift imprinted on a resonance [7]. On the other hand, d-scan variants based on third-order processes, such as third-harmonic generation (THG) $[8,9]$ and self-diffraction [10] have been demonstrated with few-cycle pulses and can in principle work in any medium, including gases. Here we demonstrate accurate on-target temporal characterization of intense hollow-core fibre (HCF) post-compressed sub-4-fs pulses using a single-beam, fully inline THG d-scan setup, where the gas target optimized for highharmonic generation (HHG) doubles as nonlinear medium, and where no additional elements are necessary other than a spectrometer.

\section{Experiment and Results}

The output pulses from a differentially-pumped HCF (pumped by $1 \mathrm{kHz}$, sub-25-fs pulses from a Ti:Sapphire amplifier at $800 \mathrm{~nm}$ ) are sent through a double-angle chirped mirror $(550-1000 \mathrm{~nm})$ and wedge compressor, resulting in $\sim 200-\mu \mathrm{J}$ pulses with a sub-4-fs Fourierlimited duration [2]. The compressed pulses are sent to a vacuum chamber and focused in a gas target (both argon and neon have been used) with a backing pressure of $\sim 1$ bar [Fig. 1(a)], producing both low-order (THG) and high-order harmonics. A movable Al mirror placed in front of the gas target is used to extract the THG signal, which is filtered and analysed with a fibre-coupled spectrometer. The THG spectrum is measured as a function of dispersion, by scanning the wedge position in the compressor around the maximum compression point, resulting in the THG d-scan trace (argon) shown in Fig. 1(b). The retrieved d-scan trace is shown in Fig. 1(c). A flip mirror placed before the entrance window of the vacuum chamber enables directing the compressed pulse to a SEA-FSPIDER setup [2], providing an independent temporal measurement.
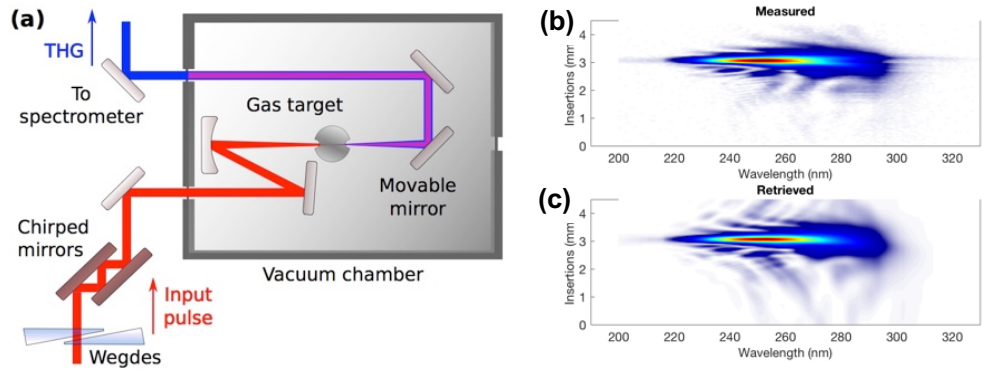

Fig. 1. (a) Setup for on-target THG d-scan and simultaneous HHG in a gas target. (b) Measured and (c) retrieved THG d-scan traces for argon.

Figure 2(a) shows the measured spectrum and the spectral phase obtained from the SEA-FSPIDER measurement (labelled as 'Reference' in Fig. 2) and the spectral phase retrieved from the THG d-scan measurement, together with its standard deviation (multiplied by a factor of 10 for clarity) obtained from 10 independent retrievals calculated with random seed phases. Figure 2(b) shows the corresponding pulses in the time domain, where an excellent agreement between SEA-F-SPIDER and d-scan is observed, down to the pre- and post-pulse structure at below $4 \%$ of the peak intensity. Measured FWHM pulse durations were $3.8 \mathrm{fs}$ for SEA-F-SPIDER and $3.9 \pm 0.1 \mathrm{fs}$ for THG d-scan. This agreement between two independent measurements of a sub-4-fs pulse, made with very different techniques based on different order nonlinear optical effects taking place in very different media (SFG in a crystal for SEA-F-SPIDER; THG in a gas for d-scan) further attests to the accuracy and 
precision of the method. Similar results were obtained in neon, although with a larger standard deviation due to the weaker nonlinearity in neon compared to argon. The measured HHG obtained in the same conditions as the THG d-scan measurement is shown in Fig. 2(c), where maximum HHG yield was observed for the compressor position corresponding to the shortest pulse. A holey mirror that transmits the HHG beam while reflecting the THG should provide a fully inline, online and on-target pulse diagnostic.
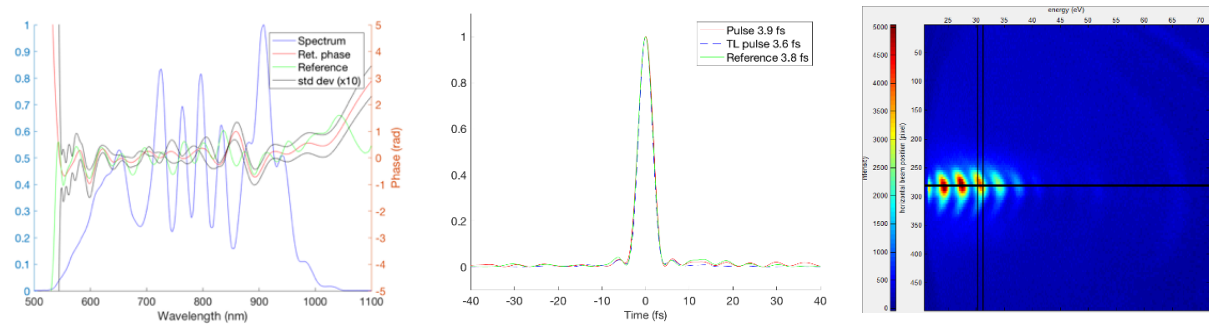

Fig. 2. (a) Spectrum and retrieved spectral phase for SEA-F-SPIDER and THG d-scan. (b) Pulse intensity profiles. (c) Spatio-spectral measurement of HHG in argon.

Acknowledgments: Fundação para a Ciência e a Tecnologia (FCT), Portugal (Grants 'UltraGraf' MERA-NET2/0002/2016, UID/ NAN/50024/2013, NORTE-01- 0145-FEDER-022096 and the Network of Extreme Conditions Laboratories - NECL).

\section{References}

1. T. Witting, F. Frank, C. A. Arrell, W. A. Okell, J. P. Marangos, and J. W. G. Tisch, Opt. Lett. 36, 1680 (2011)

2. F. Frank, C. Arrell, T. Witting, W. A. Okell, J. McKenna, J. S. Robinson, C. A. Haworth, D. Austin, H. Teng, I. A. Walmsley, J. P. Marangos, and J. W. G. Tisch, Rev. Sci. Instrum. 83, 071101 (2012)

3. M. Miranda, T. Fordell, C. Arnold, A. L'Huillier, and H. Crespo, Opt. Express 20, 688 (2012)

4. F. Silva, M. Miranda, B. Alonso, J. Rauschenberger, V. Pervak, and H. Crespo, Opt. Express 22, 10181 (2014)

5. F. Silva, B. Alonso, W. Holgado, R. Romero, J. San Román, E. Conejero Jarque, H. Koop, V. Pervak, H. Crespo, and Í. J. Sola, Opt. Lett. 43, 337-340 (2018)

6. Aart J. Verhoef, Alexander Mitrofanov, Aleksei Zheltikov, and Andrius Baltuška, Opt. Lett. 34, 82-84 (2009)

7. A. Blättermann, C. Ott, A. Kaldun, T. Ding, V. Stooß, M. Laux, M. Rebholz, and T. Pfeifer, Opt. Lett., vol. 40, 3464-3467 (2015)

8. F. Silva, M. Miranda, S. Teichmann, M. Baudish, M. Massicotte, F. Koppens, J. Biegert, and H. Crespo, in CLEO: 2013, OSA Technical Digest (online) (Optical Society of America, 2013), paper CW1H.5

9. M. Hoffmann, T. Nagy, Th. Willemsen, M. Jupé, D. Ristau and U. Morgner, Opt. Express 22, 5234 (2013)

10. M. Canhota, F. Silva, R. Weigand, and H. M. Crespo, "Inline self-diffraction dispersion-scan of over octave-spanning pulses in the single-cycle regime," Opt. Lett. 42, 3048 (2017) 